\title{
Expression of $\beta$-catenin and c-myc during human common bile duct development: a possible role in the morphogenesis of the common bile duct
}

\author{
W.L. Guo ${ }^{1}$, Q. Zhang ${ }^{2}$ and J. Wang ${ }^{2}$ \\ ${ }^{1}$ Department of Radiology, The Children's Hospital Affiliated to Soochow University, Suzhou, China \\ ${ }^{2}$ Department of General Surgery, The Children's Hospital Affiliated to Soochow University, Suzhou, China
}

\begin{abstract}
$\beta$-catenin and c-myc play important roles in the development of tissues and organs. However, little is known about their expression patterns during the development of the human common bile duct. Immunohistochemistry was used to detect $\beta$-catenin and c-myc expression in common bile duct samples from postmortem tissues of 14 premature infants and 6 spontaneously aborted fetuses. The expression of $\beta$-catenin and c-myc was also analyzed by Western blot. The samples were divided into four groups based on the stage of human fetal development: 12, 13-27, 28-37, and >37 weeks. The Image-Pro Plus v. 6.0 image analysis software was used to calculate the mean qualifying score (MQS). At fetal stages 12, 13-27, 28-37, and $>37$ weeks, MQS of $\beta$-catenin were $612.52 \pm 262.13,818.38 \pm 311.73,706.33 \pm 157.19$, and $350.69 \pm 110.19$, respectively. There was a significant difference in MQS among the four groups (ANOVA, $P=0.0155$ ) and between the scores at $>37$ and 13-27 weeks (Student-Newman-Keuls, $P<0.05$ ). At fetal stages 12, 13-27, 28-37, and $>37$ weeks, the MQS of c-myc were $1376.64 \pm 330.04,1224.18 \pm 171.66,1270.24 \pm 320.75$, and $741.04 \pm 219.19$, respectively. There was a significant difference in MQS among the four groups (ANOVA, $P=0.0087$ ) and between the scores at $>37$ and 12 weeks, $>37$ and 13-27 weeks, and $>37$ and 28-37 weeks (all $P<0.05$, Student-Newman-Keuls). Western blots showed that $\beta$-catenin and c-myc expression were significantly higher in fetal than in postnatal control duct tissue $(\mathrm{P}<0.05)$. c-myc and $\beta$-catenin are involved in the normal development of the human common bile duct.
\end{abstract}

Key words: $\beta$-catenin; c-myc; Common bile duct; Fetal development

\section{Introduction}

The Wnt/ $\beta$-catenin signaling pathway is a fundamental control mechanism in animal development and tissue homeostasis. Wnt signaling is activated during fetal development of the dorsal-ventral axis and the central nervous system in vertebrates (1). The oncoprotein $\beta$ catenin is the central regulator of gene expression in the classical Wnt signal pathway and is mainly located in the cytoplasm and may also be found in the cell membrane and nucleus. In the absence of Wnt signaling, $\beta$-catenin is phosphorylated in the cytoplasm and then degraded through the ubiquitin proteasome pathway (2). When $\beta$ catenin enters the nucleus, it specifically activates the transcription of a program of downstream target genes, including c-myc and cyclin D1, thus, controlling proliferation and differentiation (3).
During human fetal development, the endoderm close to the yolk stalk on the ventral side of the caudal foregut thickens and then forms a hollow protrusion known as the hepatic diverticulum. During weeks 3-4, the hepatic diverticulum develops into the liver, biliary system, the two ends of the main pancreatic duct, and part of the head of the pancreas. Studies have shown that the classical Wnt/ $\beta$ catenin signal pathway plays an important role in the development of the pancreas and intrahepatic duct $(4,5)$. However, research into the role of the Wnt/ $\beta$-catenin pathway in the development of the common bile duct is lacking. In this study, immunohistochemical methods and Western blots were used to examine the expression of $\beta$-catenin and its downstream target gene, c-myc, during different stages of the development of the human fetal common bile duct. 


\section{Material and Methods}

This study was approved by the Ethics Committee of the Children's Hospital Affiliated to Soochow University, and consent forms were signed by the parents in each case. In total, there were 20 fetal samples of different gestational ages (the fetal samples came from premature infants who had died in Suzhou Mudu Hospital and the Children's Hospital Affiliated to Soochow University). Fourteen samples were from premature infants and six from aborted fetuses; gestational age ranged from 12 to 42 weeks (median gestational age was 30 weeks); there were 12 males and 8 females. All deaths were natural, following abortion or premature birth. None of the cases involved pancreaticobiliary diseases.

Fetal tissues were removed within 1-6 h after death. A vertical incision was made above the rectus abdominis muscle, and skin, subcutaneous tissues, and the rectus abdominis muscle were cut to expose the abdominal cavity. After dissection, the common bile duct was removed; a portion was reserved for use in Western blotting (12 cases), and the remainder was fixed in $10 \%$ formaldehyde for $24 \mathrm{~h}$ (20 cases). Fixed tissues were embedded in paraffin, sectioned, and processed for hematoxylin and eosin or immunohistochemical staining. Common bile ducts were obtained from 9 patients (mean age 7 months, range from 4-10 months; 6 females, 3 males) during abdominal surgery for congenital choledochal cysts and used as Western blot controls.

\section{Immunohistochemical staining}

The tissues were fixed and embedded in paraffin, and serial $5-\mu \mathrm{m}$ sections were made using an LKB-V ultrathin microtome. The sections were mounted on poly-L-lysinetreated slides and heated at $60^{\circ} \mathrm{C}$ for $3 \mathrm{~h}$ before being subjected to two 10-min rounds of dewaxing using dimethylbenzene. Then, the slides were soaked in absolute ethanol, $95 \%$ ethanol, and $70 \%$ ethanol for 5 min each. The boiling hot repair method was used for antigen retrieval. Sodium citrate buffer solution (0.01 M, pH 6.0) was heated to approximately $95^{\circ} \mathrm{C}$ on an electric stove. The tissue slides were placed in the hot buffer solution for 10-15 min and then washed three times in phosphatebuffered saline (PBS). The slides were soaked in freshly prepared $0.3 \%$ peroxide-methanol blocking solution for $10 \mathrm{~min}$ at room temperature to block the activity of endogenous peroxidase. After blocking, the slides were washed three times with PBS for 5 min each. Compound digestive juice $(0.125 \%$ Trypsin $+0.1 \%$ pepsin $+0.01 \%$ EDTA) was added and the slides were incubated at $37^{\circ} \mathrm{C}$ for $20 \mathrm{~min}$ so that tissue antigens could be fully exposed. Then, $10 \%$ normal goat serum was used to block nonspecific antigens at room temperature for $10 \mathrm{~min}$, and excessive liquid was removed. A few drops of diluted primary antibodies ( $\beta$-catenin monoclonal antibody 1:100 and c-myc monoclonal antibody 1:300; ProSci Inc., USA) were added, and the slides were placed in a $4^{\circ} \mathrm{C}$ refrigerator overnight. They were rewarmed in a water bath incubator at $37^{\circ} \mathrm{C}$ for $45 \mathrm{~min}$. The slides were then rinsed three times with PBS for 5 min each. A few drops of biotin-labeled secondary antibody (Gene Tech Company Limited, China, peroxidase/diaminobenzidine, rabbit/ mouse) were added and the slides were placed in a $37^{\circ} \mathrm{C}$ water bath incubator and allowed to react for $45 \mathrm{~min}$. The slides were rinsed three times in PBS for 3 min each. A few drops of horseradish peroxidase (HRP)-labeled streptavidin were added and the slides were placed in a $37^{\circ} \mathrm{C}$ water bath incubator and allowed to react for $20 \mathrm{~min}$. The slides were then rinsed four times in PBS solution for 5 min each. The chromogenic reaction was induced using freshly prepared diaminobenzidine reagent, the time of which was adjusted under the microscope in order to adjust the degree of color change. The samples were repeatedly rinsed with distilled water to stop the reaction. The slides were then subjected to double staining with hematoxylin for 2 min, color separation with $\mathrm{HCl}$-ethanol for several seconds, bluing with ammonia water, serial alcoholic dehydration, vitrification with dimethylbenzene, and neutral resin sealing. The slides were observed under a microscope. Positive controls (extrahepatic cholangiocarcinoma) were provided by ProSci Inc. They were all positive after staining. PBS served as a negative control of immunohistochemical staining, taking the place of the primary antibody. All results were negative.

\section{Analysis of immunohistochemistry images}

Positive $\beta$-catenin staining was brown and localized in the cell membrane, cytoplasm and nucleus. Positive c-myc staining was brown and localized in the cell cytoplasm and nucleus. The samples were divided into four groups by stage (weeks) of fetal development: 12 $(n=5), 12-27 \quad(n=5), 28-37 \quad(n=5)$, and $>37$ weeks $(n=5)$. The expression levels of $\beta$-catenin and c-myc in the human fetal common bile duct at different gestational ages were systematically assessed.

Quantification of the immunohistochemical staining of $\beta$ catenin and c-myc was carried out by 2 investigators blinded to the clinical conditions using a light microscope (BX50 Olympus, Japan). For each slide, acquired images from five random fields of $400 \times$ magnification were selected for analysis by the Image-Pro Plus $v 6.0$ image analysis software (Media Cybernetics, USA). In the current study, we used a mean qualifying score (MQS) method developed and previously described by Mady and Melhem (6).

\section{Western blot}

Before examination of $\beta$-catenin and c-myc, the stored tissue was taken out of a cryogenic freezer and the weight of the common bile duct was measured by an electronic balance. For $100 \mathrm{mg}$ tissue, $1 \mathrm{~mL}$ RIPA (radioimmunoprecipitation assay) buffer and $10 \mu \mathrm{L}$ PMSF (phenylmethanesulfonyl fluoride or phenylmethylsulfonyl fluoride) were 
Table 1. Analysis of $\beta$-catenin and c-myc expression by Western blot.

\begin{tabular}{lrrrr}
\hline Groups & Cases & Mean \pm SD & Chi-square & $P$ \\
\hline$\beta$-catenin & & & & \\
$\quad$ Fetus & 12 & $3.2935 \pm 1.6746$ & & 0.0330 \\
Control & 9 & $1.7409 \pm 1.1922$ & & \\
c-myc & 12 & $1.4278 \pm 0.7479$ & 3.9596 & 0.0466 \\
Fetus & 9 & $0.8863 \pm 0.4408$ & & \\
Control & & & \\
\hline
\end{tabular}

Kruskal-Wallis test.

added. After mashing and homogenizing, the samples were centrifuged at $17,226 \mathrm{~g}$ for $30 \mathrm{~min}$ and the supernatants were collected. The protein concentrations of the supernatants were determined by a bicinchoninic acid assay (Pierce, USA). The supernatants were then mixed with a $2 \times$ loading buffer and boiled for $5 \mathrm{~min}$. The samples were used for analysis or stored at $-20^{\circ} \mathrm{C}$.

One hundred micrograms of extracted protein was subjected to SDS-PAGE. The protein was transferred to a nitrocellulose membrane. After blocking the nonspecific binding sites, a monoclonal $\beta$-catenin antibody (1:600 dilution; LifeSpan Biosciences, Inc., USA) and monoclonal c-myc antibody (1:500 dilution; LifeSpan Biosciences, Inc.) were used as the primary antibodies. After incubation at $4^{\circ} \mathrm{C}$ overnight, an HRP-labeled secondary antibody was added. The bands were detected with an enhanced chemiluminescence reagent for $1 \mathrm{~min}$. The quantitative analysis of $\beta$ catenin and c-myc was performed by normalizing against GAPDH using analysis software (Tanon-1600, China).

\section{Statistical analysis}

All data were processed by the SAS v8.1 software (SAS Institute Inc., USA) and are reported as means $\pm S D$. Based on the data distribution and variance homogeneity test, one-way ANOVA, Student-NewmannKeuls, or Kruskal-Wallis tests were used to compare different groups. A $P$ value $<0.05$ was considered to be statistically significant.

\section{Results}

$\beta$-catenin and c-myc expression by Western blot

As shown in Table 1 and Figure 1, $\beta$-catenin expression

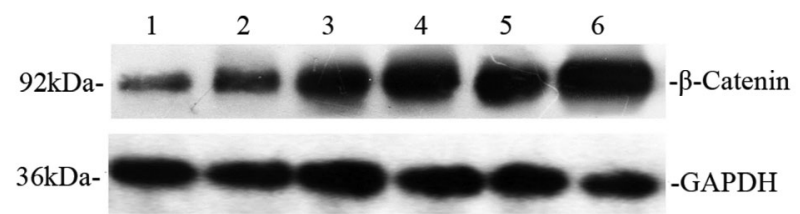

Figure 1. Expression of $\beta$-catenin was significantly higher in the fetus group (lanes $4,5,6$ ) than in the control group (lanes $1,2,3)$ by Western blot. was $3.2935 \pm 1.6746$ in the fetus group, which was significantly higher than in the control group (1.7409 \pm $1.1922 ; \mathrm{P}<0.05)$. The expression of $\mathrm{c}-\mathrm{myc}$ was $1.4278 \pm 0.7479$ in the fetus group, which was also significantly higher than in the control group $(0.8863 \pm$ 0.4408; $\mathrm{P}<0.05$; Table 1 and Figure 2).

\section{$\beta$-catenin and c-myc expression by immunohistochemistry}

Positive $\beta$-catenin expression in the common bile duct was indicated by brown staining. In 12-week fetuses, $\beta$ catenin expression was seen in the cell membranes of cylindrical epithelial cells, accompanied by some expression in the cytoplasm and nucleus. $\beta$-catenin expression was also detected in the cytoplasm and cell membranes of cells of the plexus, but only nuclear expression was detected in interstitial cells. In 13-27-week samples, $\beta$ catenin expression was observed in the cell membrane, cytoplasm, and nucleus in the cylindrical epithelium, the cytoplasm and cell membrane in the plexus, and in the nucleus in interstitial cells. At 28-37 weeks, $\beta$-catenin expression was observed in the cell membranes of the cylindrical epithelium cells accompanied by some cytoplasmic and nuclear staining. Staining of the cytoplasm and cell membrane was visible in the plexus, but only nuclear staining was seen in interstitial cells. After 37 weeks of fetal development, $\beta$-catenin expression was observed in the cell membrane and nucleus in both the cylindrical epithelium and the plexus (Figure 3 ). At fetal stages 12 , $13-27,28-37$, and $>37$ weeks, the MQS of $\beta$-catenin were $612.52 \pm 262.13,818.38 \pm 311.73,706.33 \pm 157.19$, and $350.69 \pm 110.19$, respectively. There was a significant difference in MQS among the four groups (ANOVA, $P=0.0155)$ and between the scores at $>37$ and 13-27 weeks (Student-Newman-Keuls, $\mathrm{P}<0.05$ ), but there were no significant differences between the other groups.

Positive c-myc expression was observed at all stages of human fetal development, mostly in the cytoplasm and nucleus (Figure 4). At fetal stage 12, 13-27, 28-37, and $>37$ weeks, the MQS of c-myc were $1376.64 \pm 330.04$ $1224.18 \pm 171.66,1270.24 \pm 320.75$, and $741.04 \pm 219.19$, respectively. There was a significant difference in MQS among the four groups (ANOVA, $\mathrm{P}=0.0087$ ) and between 


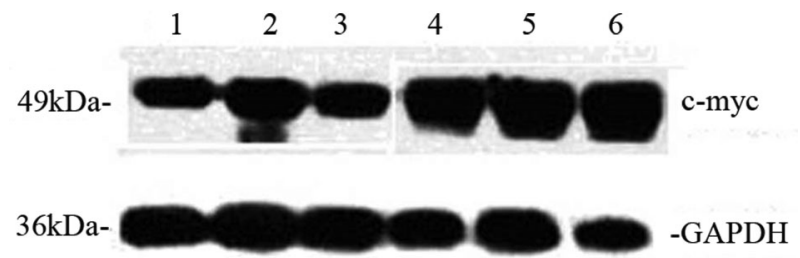

Figure 2. Expression of c-myc was significantly higher in the fetus group (lanes 4,5,6) than in the control group (lanes 1,2,3) by Western blot.

scores at $>37$ and 12 weeks, $>37$ and 13-27 weeks, and $>37$ and 28-37 weeks (all $\mathrm{P}<0.05$, Student-NewmanKeuls). There were no significant differences between the other groups.

\section{Discussion}

The protein $\beta$-catenin is encoded by a proto-oncogene. Its function is highly conserved across animal evolution. It has two key functions in metazoan organisms. First, it binds to E-cadherin to form the E-cadherin- $\beta$-catenin functional complex, which is a key structure of the cell adhesion junction. It controls the adhesion and motility of cells and regulates the structural integrity and morphogenesis of tissues. Second, it is a key component of the canonical Wnt signal transduction pathway. The Wnt signal pathway participates in many fetal development processes, such as the development of the dorsal-ventral axis and the central nervous system in vertebrate fetuses. The Wnt signal transduction pathway is usually turned off in normal mature cells (1). Early defects in the Wnt signal transduction pathway can cause many development problems.

The results of this study show that $\beta$-catenin is highly expressed in fetuses before 37 weeks. In fetuses at 12

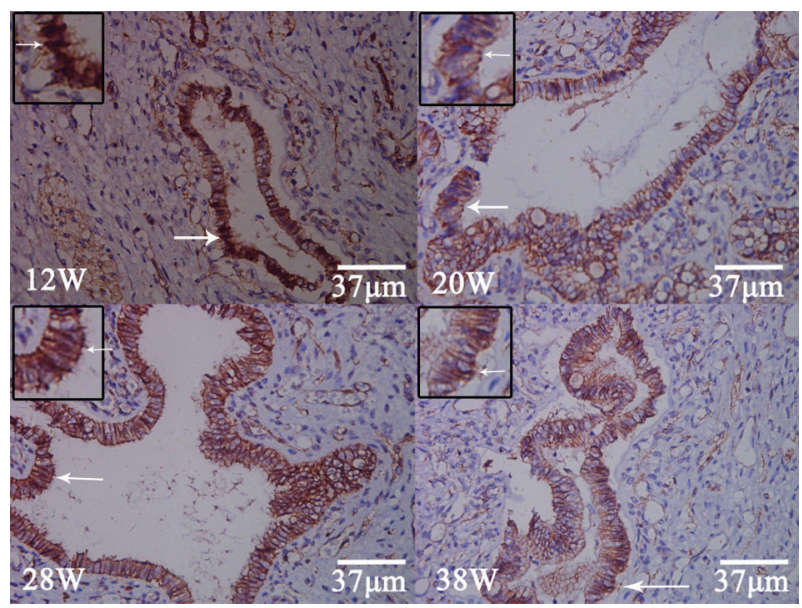

Figure 3. Positive expression of $\beta$-catenin (arrows) in the common bile duct cells in the membrane, cytoplasm and nucleus at different fetal stages $(12,20,28$, and 38 weeks).

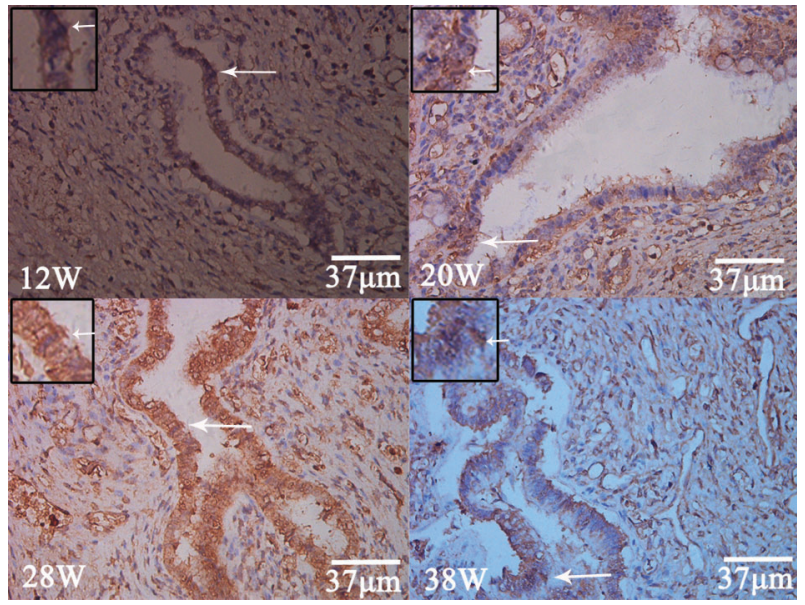

Figure 4. Positive expression of c-myc (arrows) in the common bile duct cells in the cytoplasm and nucleus at different fetal stages $(12,20,28$, and 38 weeks).

weeks of development and younger, $\beta$-catenin was highly expressed, mostly in the cell membrane but also in the cytoplasm and nucleus. At 13-27 weeks, $\beta$-catenin was highly expressed, mostly in the cell membrane, accompanied by expression in the cytoplasm and nucleus. At 28-37 weeks, $\beta$-catenin expression remained high, similar to that seen at 13-27 weeks. During this time, it is possible that the activation of Wnt signaling causes $\beta$-catenin proteins to accumulate and then enter cell nuclei, where they bind to lymphoid enhancer factor/T-cell factor (Lef/ Tcf) and activate the transcription of downstream target genes (7). However, after 37 weeks of fetal development, $\beta$-catenin expression was lower than at earlier stages. At this time, it was mostly expressed in the cell membrane, although some nuclear expression was also detected. Because the majority of the membrane $\beta$-catenin proteins reside in adhesion junctions, it may be that during the stage of $>37$ weeks, the structures of common bile duct tissue become stabilized by the formation of intercellular junctions, and that the common bile duct matures during this stage. However, before 37 weeks, $\beta$-catenin is expressed in the cell membrane as well as in the cytoplasm and nucleus. The $\beta$-catenin in the cytoplasm and nucleus may play a role in regulating the development of the common bile duct through the classical Wnt signal transduction pathway. Western blots also showed that the expression of $\beta$-catenin was higher than in control tissues.

A study with transgenic mice by Tan et al. (8) found that $\beta$-catenin overexpression causes increased proliferation of liver cells and an increase in the size of the liver. The results of the current study showed that during weeks 13-27 of fetal development, $\beta$-catenin expression was the highest. This suggested that at 13-27 weeks, the common bile duct cells are at a stage of substantial proliferation. Hussain et al. (9) cultured mouse fetal liver cells and, at 10 days of development, found that Wnt signaling 
significantly affected the differentiation of intrahepatic bile duct cells and the transformation of liver cells. An immunohistochemical study of 31 cases of $\beta$-catenin expression in the intrahepatic bile duct during different fetal development stages by Terada et al. (5) found that $\beta$ catenin expression was observed throughout the development of the bile duct (ductal plate, bile duct reformation, and immature bile duct). During bile duct reformation and the immature bile duct stage, $\beta$-catenin expression was high, localized mainly in the cell membrane. These results suggest that $\beta$-catenin is involved in the development of the fetal intrahepatic bile duct.

c-myc is an important target gene in the classical Wnt/ $\beta$-catenin signal pathway (10). It participates in cell growth, proliferation, differentiation, and apoptosis and is involved in the development of many types of tumors. Abnormal activation of Wnt signals can induce cell proliferation and the development of tumors. In the promoter region of human c-myc, there is an Lef/Tcfresponse element called TBE3. $\beta$-catenin binds to TBE3 and activates the transcription of c-myc.

In our data, c-myc positive staining was observed at all stages of human fetal development that were evaluated. Before 37 weeks, c-myc expression was high, mainly in the cytoplasm and nuclei. However, after 37 weeks, the expression was dramatically reduced to levels lower than observed at earlier stages. Changes in c-myc expression were similar to those of $\beta$-catenin. This suggests that, before 37 weeks, the common bile duct is at a stage of relatively strong proliferation, so a relatively large amount of c-myc accumulates in the cytoplasm and nuclei. However, after 37 weeks, the relative low expression of c-myc at that time suggests the common bile duct has entered a relatively mature stage, and the proliferation rate is decreased. Western blots also showed that the expression of c-myc was higher in the fetus group than in the control group. Using RNA in situ hybridization, Schmid and Schulz (11) detected c-myc, alpha-fetoprotein, and albumin in the livers of fetal mice, and they found that when c-myc was highly expressed in the fetal liver, liver precursor cells proliferated and aggregated very quickly,

\section{References}

1. Bellipanni G, Varga M, Maegawa S, Imai Y, Kelly C, Myers $A P$, et al. Essential and opposing roles of zebrafish betacatenins in the formation of dorsal axial structures and neurectoderm. Development 2006; 133: 1299-1309, doi: 10.1242/dev.02295.

2. Taurin S, Sandbo N, Qin Y, Browning D, Dulin NO. Phosphorylation of beta-catenin by cyclic AMP-dependent protein kinase. J Biol Chem 2006; 281: 9971-9976, doi: 10.1074/jbc.M508778200.

3. Klaus A, Birchmeier W. Wnt signalling and its impact on development and cancer. Nat Rev Cancer 2008; 8: 387398, doi: $10.1038 /$ nrc2389. differentiating toward a specific stage. This suggests that c-myc plays an important role in the development of the fetal liver. Wang et al. (12) used immunohistochemistry, Western blotting, and RT-PCR to examine the role of c-myc in the development of fetal mouse hair follicle. They directly demonstrated that c-myc promoted the proliferation of hair keratinocytes and the differentiation of inner root sheaths. Cohen et al. (13) found c-myc to be vital for the expansion of fetal alveoli and intestinal mucosa tissues. The role of cmyc in the embryogenesis of mice has also been reported (14). Nakhai et al. (15) found Wnt/ $\beta$-catenin signaling to regulate the development of pancreas epithelial cells and promote the proliferation of pancreatic acinar cells via its target gene c-myc. All of those studies demonstrate the importance of c-myc in fetal development. The results of the current study show that c-myc is expressed during all stages of development of the human fetal common bile duct, but at 12 weeks, its expression pattern does not completely match that of $\beta$-catenin, suggesting that c-myc expression is not necessarily controlled solely by the Wnt/ $\beta$-catenin signaling pathway but may also be regulated by other pathways, such as the Notch or Hedgehog pathways $(16,17)$. Further investigation is required to determine the exact signal pathways that control c-myc and promote proliferation.

In summary, immunohistochemistry and Western blot were used to examine the expression of $c-m y c$ and $\beta$ catenin in the human common bile duct during different stages of fetal development. Results showed that c-myc and $\beta$-catenin were involved in the development of the human common bile duct. They also suggested that the classical Wnt/ $\beta$-catenin signaling pathway may play an important role in the development of the common bile duct.

\section{Acknowledgments}

Research supported by the Jiangsu Province Natural Science Research Projects in Colleges and Universities (\#13KJB320020). The study was also funded by Jiangsu Province "333" Talents.

4. Heiser PW, Lau J, Taketo MM, Herrera PL, Hebrok M. Stabilization of beta-catenin impacts pancreas growth Development 2006; 133: 2023-2032, doi: 10.1242/dev. 02366 .

5. Terada T, Ashida K, Kitamura Y, Matsunaga Y, Takashima K, Kato M, et al. Expression of epithelial-cadherin, alpha-catenin and beta-catenin during human intrahepatic bile duct development: a possible role in bile duct morphogenesis. $J$ Hepatol 1998; 28: 263-269, doi: 10.1016/0168-8278(88)80013-8.

6. Mady HH, Melhem MF. FHIT protein expression and its relation to apoptosis, tumor histologic grade and prognosis in colorectal adenocarcinoma: an immunohistochemical and 
image analysis study. Clin Exp Metastasis 2002; 19: 351358, doi: 10.1023/A:1015594702522.

7. Torre C, Perret C, Colnot S. Transcription dynamics in a physiological process: beta-catenin signaling directs liver metabolic zonation. Int J Biochem Cell Biol 2011; 43: 271278.

8. Tan X, Apte U, Micsenyi A, Kotsagrelos E, Luo JH, Ranganathan S, et al. Epidermal growth factor receptor: a novel target of the Wnt/beta-catenin pathway in liver. Gastroenterology 2005; 129: 285-302, doi: 10.1053/j.gastro. 2005.04.013.

9. Hussain SZ, Sneddon T, Tan X, Micsenyi A, Michalopoulos GK, Monga SP. Wnt impacts growth and differentiation in ex vivo liver development. Exp Cell Res 2004; 292: 157-169, doi: 10.1016/j.yexcr.2003.08.020.

10. Roh MS, Hong SH, Jeong JS, Kwon HC, Kim MC, Cho SH, et al. Gene expression profiling of breast cancers with emphasis of beta-catenin regulation. J Korean Med Sci 2004; 19: 275-282, doi: 10.3346/jkms.2004.19.2.275.

11. Schmid P, Schulz WA. Coexpression of the c-myc protooncogene with alpha-fetoprotein and albumin in fetal mouse liver. Differentiation 1990; 45: 96-102, doi: 10.1111/j.14320436.1990.tb00462.x.

12. Wang N, Yang T, Li J, Lei M, Shi J, Qiu W, et al. The expression and role of c-Myc in mouse hair follicle morphogenesis and cycling. Acta Histochem 2012; 114 : 199-206, doi: 10.1016/j.acthis.2011.04.009.

13. Cohen JC, Scott DK, Miller J, Zhang J, Zhou P, Larson JE. Transient in utero knockout (TIUKO) of C-MYC affects late lung and intestinal development in the mouse. BMC Dev Biol 2004; 4: 4, doi: 10.1186/1471-213X-4-4.

14. Naz RK, Kumar G, Minhas BS. Expression and role of cmyc protooncogene in murine preimplantation embryonic development. J Assist Reprod Genet 1994; 11: 208-216, doi: 10.1007/BF02211810.

15. Nakhai H, Siveke JT, Mendoza-Torres L, Schmid RM. Conditional inactivation of Myc impairs development of the exocrine pancreas. Development 2008; 135: 3191-3196, doi: $10.1242 /$ dev. 017137

16. Klinakis A, Szabolcs M, Politi K, Kiaris H, Artavanis-Tsakonas $\mathrm{S}$, Efstratiadis A. Myc is a Notch1 transcriptional target and a requisite for Notch1-induced mammary tumorigenesis in mice. Proc Natl Acad Sci U S A 2006; 103: 9262-9267, doi: 10.1073/ pnas.0603371103.

17. Sicklick JK, Li YX, Jayaraman A, Kannangai R, Qi Y, Vivekanandan $P$, et al. Dysregulation of the Hedgehog pathway in human hepatocarcinogenesis. Carcinogenesis 2006; 27: 748-757, doi: 10.1093/carcin/bgi292. 